\title{
Revisión sistemática de literatura. Declinación funcional en ancianos hospitalizados
}

\author{
José Mauricio Ocampo, MD, $\mathrm{MSc}^{1,2}$, Carlos A. Reyes-Ortiz, $\mathrm{MD}, \mathrm{PhD}^{3}$ \\ 1 Profesor Asistente, Departamento de Medicina Familiar, Universidad del Valle, Cali, Colombia. \\ 2 Profesor Asociado, Departamento de Medicina Interna, Universidad Libre, Cali, Colombia. \\ 3 Division of Geriatric and Palliative Medicine, Department of Internal Medicine, University of Texas Medical School at Houston, USA. \\ E-mail: careyesortiz@hotmail.com
}

Fecha de Recepción: 22/11/2015

Fecha de Evaluación: 24/2/2016

Fecha de Solicitud de Correcciones: 3/3/2016

Fecha de Aceptación: 7/3/2016

\section{Resumen}

Los ancianos requieren con frecuencia admisión hospitalaria, durante la cual se generan riesgos para desarrollar eventos adversos, que en ocasiones no se relacionan con las características del anciano ni la enfermedad aguda, sino con tratamientos médicos, el ambiente y las rutinas de cuidado hospitalario. Los ancianos con enfermedad aguda son un grupo heterogéneo y vulnerable debido a múltiples compromisos en las esferas biopsicosociales como disminución de la reserva funcional, comorbilidad, polifarmacia, alteraciones cognoscitivas, compromiso funcional y alteraciones en las esferas social y familiar. Estas características ubican al anciano en riesgo para desarrollar durante la hospitalización declinación funcional, por lo cual se debe realizar identificación oportuna de los factores de riesgo con el objetivo de prevenir y manejar esta condición mediante un equipo interdisciplinario. La implementación de modelos de atención que prevengan y mejoren el compromiso funcional deber ser una prioridad para las instituciones y personal de salud que atienden ancianos. Estos modelos incluyen revisión del manejo farmacológico, rehabilitación interdisciplinaria temprana, programas de ejercicio y diseño del egreso geriátrico planeado.

Palabras clave: Anciano; Hospitalización; Evaluación Geriátrica

\section{Functional decline in hospitalized elderly}

Abstract

The elderly require frequent hospital admission. There are greater risks for adverse outcomes during hospitalization. Those events are not always associated with the characteristics of the elderly or acute disease, but with medical treatments, the environment and routine hospital care. Older persons with acute disease are heterogeneous and vulnerable groups because they have many bio psychosocial changes including decreased functional reserve, high comorbidity, polypharmacy, cognitive impairment, functional impairment and alterations in the social and familiar areas. These features put the elderly at greater risk for developing adverse events related to hospitalization such as functional decline. Wherefore it should be performed timely identification of the risk factors for functional decline in order to prevent and manage this condition by an interdisciplinary team. The implementation of models of care to prevent and improve functional compromise should be a priority for institutions and health personnel serving the elderly. These models include review of pharmacological management, early interdisciplinary rehabilitation, exercise programs and design of a geriatric planned discharge.

Keywords: Aged; Geriatric assessment; Hospitalization.

\section{Introducción}

El fenómeno de la transición demográfica ha generado un incremento en la prevalencia de enfermedades crónicas y por consiguiente aumento en dependencia y discapacidad. Debido al deterioro del estado de salud y compromiso funcional, los ancianos requieren con mayor frecuencia de atención en salud, lo cual es evidente en los servicios hospitalarios.

Los ancianos que requieren de atención hospitalaria se caracterizan por presentar una baja reserva fisiológica y múltiples comorbilidades, lo cual puede incrementar el riesgo para desarrollar desenlaces adversos en salud relacionados con la hospitalización, que en ocasiones no se asocian con las características del anciano ni la enfermedad aguda, sino con tratamientos médicos, el medio y las rutinas de cuidado hospitalario (1). Los ancianos agudamente enfermos se caracterizan por ser un grupo heterogéneo y vulnerable debido a múltiples compromisos en las esferas biopsicosociales como edad avanzada, disminución de reserva funcional, múltiples enfermedades crónicas, polifarmacia, alteraciones cognoscitivas, compromiso funcional y baja red de apoyo social y familiar. Estas características están asociadas con mayor riesgo para desarrollar declinación funcional (DF), como producto de la carga acumulada de envejecimiento, fragilidad y enfermedad (1).

En la actualidad los servicios hospitalarios experimentan presión asistencial por la carencia histórica de infraestructura, dotación tecnológica y recursos humanos, así como mayor demanda de atención de ancianos, que requieren mayores cuidados por su condición de vulnerabilidad y fragilidad (2).

Lo anterior produce una situación paradójica, dado que aunque se tienen muchos hospitales modernos con adecuada tecnología, éstos se encuentran diseñados para pacientes jóvenes o adultos con enfermedad aguda, a pesar que el mayor número de enfermos que ocupan estas camas son ancianos frágiles, vulnerables y con múltiples comorbilidades, lo cual hace que se requieran condiciones de atención diferentes a las ofrecidas en la actualidad (2).

Asimismo, muchas de las prácticas de cuidado hospitalario son inadecuadas dado que se fundamentan más en la tradición y experiencia que en la evidencia científica, por ejemplo, promueven el reposo en cama lo cual puede generar desenlaces adversos como desacondicionamiento físico, síndrome de inmovilidad, neumonía y embolismo pulmonar entre otros (2). 
Este escenario la hospitalización se convierte en una situación de riesgo para el estado de salud y funcional del anciano. El cuidado hospitalario usual se orienta solo al manejo de condiciones médicas agudas, sin prestar atención en la mayoría de las situaciones al estado cognoscitivo, social ni funcional; en consecuencia, durante ese tiempo, existe un alto riesgo para DF. Por ejemplo, las indicaciones de movilización temprana son instauradas solo cuando el paciente está próximo al egreso, ya cuando puede ser muy tarde sin tener presente que la DF se desarrolla tan rápido como desde el segundo día de hospitalización (3).

Con frecuencia los ancianos egresan una vez resuelta la condición médica aguda que generó la hospitalización, sin prestar atención a la condición funcional. Por consiguiente, en un alto porcentaje de ancianos, el estado funcional al alta no es el mismo previo a la hospitalización y en la mayoría de los casos desarrollan DF, comprometiéndose tanto las actividades básicas de la vida diaria (ABVD) como actividades instrumentales de la vida diaria (AIVD) (4).

\section{Definición}

Se han empleado diferentes términos para describir la DF como la pérdida de la función o independencia, declinación en las ABVD o AIVD, estado declinante y alteración funcional. El término que se empleará en adelante será DF. La definición de mayor uso en la literatura hace mención a la pérdida de la capacidad para realizar al menos una ABVD necesarias para vivir de forma independiente y sin asistencia al alta hospitalaria, que no presentaban antes del inicio de la enfermedad aguda. Se compara la condición funcional basal dos semanas antes del inicio de la enfermedad aguda, con el momento del ingreso y el alta hospitalaria (5). Los cambios funcionales presentes durante el período del ingreso son un proceso dinámico que tienen diferentes transiciones, incluye desde el declinar previo al mismo, pasando por la recuperación o la progresiva declinación durante la hospitalización y finaliza con la evolución en los meses siguientes al alta (6).

La DF asociada con la hospitalización se compone y caracteriza por tener las siguientes trayectorias $(4,6,7)$ :

Deterioro previo a la hospitalización. Son pacientes que presentan DF desde el inicio del proceso agudo de la enfermedad previo a la hospitalización, es el más frecuente y refleja la severidad de la enfermedad. En la mayoría de los estudios se valora dos semanas antes del
Figura 1. Trayectorias funcionales en ancianos hospitalizados por enfermedad aguda. Pacientes que ingresan sin DF respecto a su situación basal (a). Algunos son dados de alta del hospital sin DF (b), otros se deterioran en el ingreso (c). Pacientes que ingresan con DF respecto a su situación basal (d), pueden recuperarse durante la hospitalización (e), no mejorar (f) o incluso empeorar (g). En todos los casos, habrá posibilidad de recuperarse total o parcialmente si se adoptan a tiempo las medidas adecuadas (flechas discontinuas). Se muestra también la DF hospitalaria global y el curso de la DF.

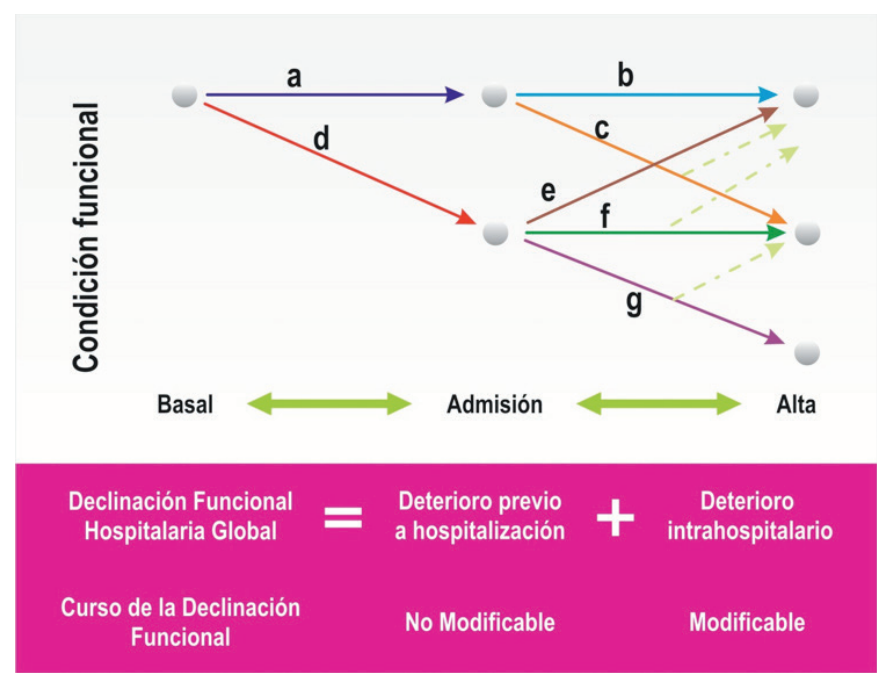

Modificada de Covinsky et al..$^{4}$, Osuna-Pozo et al. ${ }^{5}$

inicio de la enfermedad aguda y es poco susceptible de ser modificado. Deterioro intrahospitalario. Son pacientes que al ingreso hospitalario no presentan cambios en su estado funcional, no obstante, durante el tiempo de hospitalización puede comprometerse. Es susceptible de ser modificada durante el período de hospitalización pudiendo recuperar parcial o totalmente si se realizan las intervenciones adecuadas.

Declinación funcional global. Está compuesta por el deterioro previo a la hospitalización y el deterioro intrahospitalario (Figura 1).

\section{Epidemiología}

Las investigaciones realizadas muestran una marcada variabilidad en la prevalencia de DF asociada con hospitalización, probablemente explicado por diferencias en características de la población estudiada (edad, comorbilidad, estado cognoscitivo, condición funcional de base entre otras), metodología empleada para definir y evaluar la DF y los servicios de hospitalización estudiados (urgencias, servicios de hospitalización convencionales o unidades geriátricas).

Existen varios trabajos clásicos, dentro de los cuales figuran Covinsky et al. (4) y Sager et al. (8), realizados para determinar la modificación del estado funcional de pacientes mayores de 70 años admitidos en servicios de hospitalización de medicina interna, y documentaron que $31 \%$ y $35 \%$ de los pacientes desarrollaron DF al alta. Hallazgos similares se encontraron en el estudio HOPE (9), donde 40\% de los ancianos que presentaron $\mathrm{DF}$ al egreso tuvieron compromiso en $3 \mathrm{o}$ más ABVD.

Según el tipo de unidad hospitalaria, la frecuencia de DF varía. Por ejemplo, en el estudio de Abizanda et al. realizado en ancianos 
frágiles atendidos en una unidad geriátrica de agudos (UGA) 83,7\% desarrollaron DF (10).

Zelada et al. realizaron una investigación para comparar la frecuencia de DF en una Unidad Geriátrica de Agudos (UGA) con una unidad de hospitalización convencional; 19,1\% desarrolló DF en la UGA, comparado con el $40 \%$ en la unidad convencional (11).

Con respecto a la evolución en el tiempo del estado funcional posterior al egreso hospitalario, se tiene que los ancianos que desarrollan DF tienen mayor probabilidad de no mejorar e incluso empeorar la condición funcional, ser institucionalizados y morir, comparado con el grupo que no presentó DF (6). Por ejemplo, Boyd et al. (6), realizó seguimiento durante un año posterior al egreso, y encontró que el compromiso para realizar las ABVD en el grupo de ancianos con DF fue del $28,6 \%$, a diferencia del $15,2 \%$ en el grupo que no había desarrollado DF.

Modelo conceptual de declinación funcional

Figura 2. Modelo conceptual de la declinación funcional. Adaptada de Verbrugge y Jette ${ }^{12}$

\section{Vía Principal}

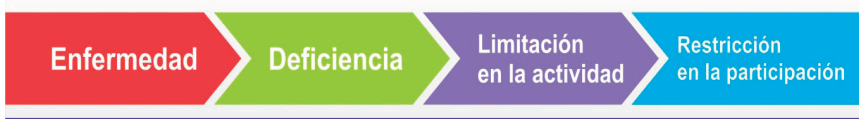

\section{Severidad de la enfermedad aguda}

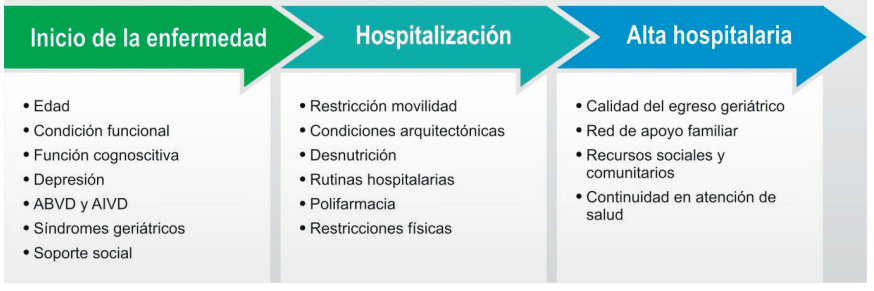

El modelo conceptual empleado para explicar el proceso de DF asociada con hospitalización se fundamenta en el descrito por Verbruege y Jette (12); también se tomaron elementos del modelo de Nagi. (13). El modelo de Verbruege y Jette presenta gran capacidad de adaptación a distintos enfoques y es el más empleado para la investigación en discapacidad (12). Según los autores, la discapacidad es el resultado final del camino que va desde las alteraciones en salud hasta las pérdidas funcionales (Figura 2). Está compuesto por una vía central donde se describen las transiciones desde la aparición de enfermedad, la deficiencia que resulta en algún sistema u órgano, limitaciones en el funcionamiento que derivan de estas deficiencias y por último la expresión de limitaciones funcionales y discapacidad para llevar a cabo las ABVD y AIVD. La discapacidad se suele operacionalizar según el grado de dificultad para realizar las ABVD para el cuidado e higiene personal y AIVD para el mantenimiento del hogar y relación con el mundo externo.

Tanto Verbrugge y Jette (12) como Nagi (13) al describir las trayectorias desde la enfermedad de origen hasta la discapacidad, introducen factores que aceleran o lentifican el recorrido a través de este trayecto, mostrando que este proceso no se produce de forma aislada, ni es inalterable. Por consiguiente, existen factores sociales y de contexto que la pueden alterar o modular en un sentido u otro.
El proceso de la DF está influido por el entorno del anciano (cuidados médicos, regímenes terapéuticos, condiciones socioeconómicas, redes sociales, ayudas técnicas y características del medio hospitalario) y por recursos internos ante las nuevas situaciones de salud (autopercepción de salud, control interno, situación económica y estrato social) $(14,15)$. Este proceso está condicionado por lo que se ha experimentado a lo largo de la vida y que ha podido influir en la salud.

Dentro de los factores extraindividuales del modelo en un anciano que requiere ser hospitalizado se encuentran las características locativas del medio hospitalario, dado que muchas de estas instituciones fueron diseñadas estructuralmente para suplir más las necesidades del personal de salud que para los pacientes, sin promover el autocuidado y funcionalidad en los ancianos (16).

El proceso de DF es dinámico, con diferentes trayectorias que se presentan antes, durante y después de la hospitalización. De esta manera se observan deterioros y/o mejorías de la capacidad funcional a lo largo del tiempo. Los cambios dependen del medio externo y de las características biopsicosociales del anciano, así como de condiciones que han determinado la exposición a factores de riesgo y protectores para la salud a lo largo de la vida.

Por ejemplo, habitaciones y corredores con obstáculos y poco iluminados desestimulan la deambulación e incrementan el riesgo de caídas; el trato poco familiar y despersonalizado llevan a restringir la autonomía del paciente. De esta forma los factores extraindividuales pueden afectar los factores intraindividuales generando DF; por ejemplo, el desarrollo de inmovilidad puede ser potenciado por órdenes médicas de reposo en cama sin clara indicación, llevando a pérdida del tono vascular, desacondicionamiento y sarcopenia. Además, la inmovilidad favorece el desarrollo de hipotensión postural y úlceras por presión (17).

La combinación de factores intra y extraindividuales pueden potenciar la DF, tal como ocurre en situaciones donde se limita la ingesta de alimentos debido a procedimientos diagnósticos o terapéuticos como endoscopias o estudios imagenológicos, lo cual ocasiona modificaciones en la dieta o períodos de ayuno prolongado (18).

Algo semejante ocurre con los medicamentos, aunque son esenciales para el cuidado del paciente, si se prescriben de forma inapropiada pueden generar eventos adversos como delirium y potenciar deterioro cognoscitivo en pacientes con demencia de base (19).

\section{Consecuencias de la declinación funcional}

Desarrollar DF durante la hospitalización puede producir consecuencias que suelen ser severas y en ocasiones de mayor gravedad cuando se compara con la enfermedad aguda que generó la hospitalización (20).

Durante la hospitalización los ancianos que desarrollan DF presentan peor evolución clínica y mayor número de complicaciones, estancia hospitalaria, costos económicos sociales, consumo de recursos y reingresos (21), además se asocia con mayor probabilidad de muerte posterior al egreso (22). También la DF se relaciona con mayor probabilidad de ingreso a servicios hospitalarios de larga estancia (23) e institucionalización (24). Aunque la decisión de ingreso a estas instituciones no solo depende de la condición funcional sino de factores culturales, económicos, sociales como el vivir solo, las preferencias del paciente y su familia (24). 


\section{Fisiopatología de la declinación funcional}

La fisiología normal del envejecimiento produce cambios que disminuyen la reserva fisiológica, que en condiciones normales y sin enfermedad le permiten al anciano funcionar sin dificultad. Sin embargo durante la hospitalización hay mayor riesgo para desarrollar DF, porque además de los cambios fisiológicos del envejecimiento se sobreponen múltiples factores de estrés como la enfermedad aguda, medicamentos y rutinas hospitalarias.

Dentro de los cambios fisiológicos relacionados con el envejecimiento que incrementan el riesgo para desarrollar DF se tiene la disminución de la masa y fuerza muscular, baja densidad mineral ósea, disminución del agua corporal total, volumen plasmático y sanguíneo, capacidad aeróbica y ventilatoria, inestabilidad vasomotora en barorreceptores, disminución en la sensación de sed, gusto, olfato, dentición y la fragilidad cutánea (17).

Con respecto a los factores relacionados con la hospitalización, se encuentran el reposo en cama que genera efectos deletéreos como disminución de la movilidad (por enfermedad aguda, empleo de inmovilizaciones físicas y/o químicas), aislamiento sensorial y social, dietas restrictivas, polifarmacia, medio y rutinas hospitalarias (17).

Figura 3. Fisiopatología de la declinación funcional asociada con hospitalización. Modificada de Osuna-Pozo et al. ${ }^{5}$ y Lafont et al. ${ }^{29}$

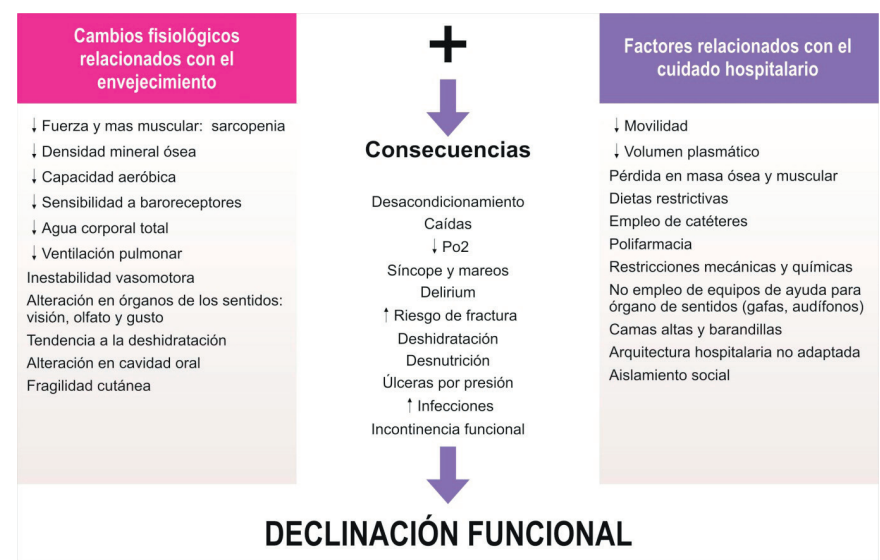

Una vez el anciano desarrolla la enfermedad aguda, los cambios asociados con el envejecimiento y los factores relacionados con la hospitalización tienen efecto sinérgico pudiendo desencadenar "la cascada de la dependencia", como son mayor compromiso de la fuerza muscular en particular de miembros inferiores que altera la movilidad y marcha, disminución en la capacidad aeróbica, desarrollo de contracturas, úlceras por presión, osteopenia acelerada, sarcopenia y progresión de la fragilidad, las cuales se potencian entre sí, dan lugar a la aparición de múltiples problemas de salud, síndromes geriátricos y generando como resultado final DF (Figura 3) $(5,17)$. La DF relacionada con la hospitalización puede ser explicada a través del paradigma de los síndromes geriátricos, como caídas, delirium e incontinencia $(25,26)$.

Como en los síndromes geriátricos, la DF rara vez puede ser explicada por una sola causa, se presenta en un anciano vulnerable con déficits en múltiples sistemas orgánicos que se hacen evidentes en situaciones de estrés como la enfermedad aguda y la hospitalización, que constituyen factores precipitantes para desarrollar DF (4). La DF comparte un proceso común con los síndromes geriátricos que incluye la interacción entre la condición de vulnerabilidad de base y capacidad de recuperación (resiliencia), la severidad del factor precipitante (enfermedad aguda) y el proceso de atención (cuidado hospitalario) que pueden afectar la recuperación funcional y promover DF (20).

\section{Factores de riesgo de la declinación funcional}

La identificación de los factores de riego (FR) asociados con DF permitirá prevenirlos y corregirlos, aunque la gran mayoría están bien detectados, existen otros por determinar y estudiar.

Existen varias revisiones donde muestran que los principales FR para DF son la edad, el diagnóstico de ingreso, la condición funcional antes de hospitalización, el deterioro cognoscitivo, el delirium y la depresión $(20,27,28)$. Últimamente la condición de fragilidad ha surgido como un factor que ha ganado importancia en el contexto de DF (29).

Inmovilidad. Debido a los cambios fisiológicos con el envejecimiento que disminuyen la reserva muscular, la condición de inmovilidad presente durante la hospitalización tiene un efecto potenciador para la DF. La inmovilidad lleva a rápida pérdida de masa muscular, disminuye la síntesis de proteínas musculares, produce cambios en articulaciones generando dolor, alterando la movilidad y capacidad aeróbica (30).

Durante la hospitalización los ancianos tienen poca movilidad, documentándose que entre el $73 \%$ y $83 \%$ del tiempo permanecen en cama (31). La pérdida de masa muscular en las extremidades inferiores, determina la condición funcional del anciano al comprometer la movilidad y marcha, aparece de forma temprana, siendo evidentes después de los primeros dos días de reposo en cama (31). Para mantener la fuerza muscular se requiere de contracciones musculares, porque la fuerza muscular puede disminuir hasta $5 \%$ por día (30). Los músculos antigravitatorios de las piernas y espalda son los responsables para mantener la postura erguida y los que se afectan durante períodos de inmovilidad. Las hospitalizaciones recurrentes pueden llevar a un ciclo de atrofia y recuperación muscular con DF. En los ancianos por sus cambios fisiológicos el proceso de restauración de la masa muscular es menos efectivo, lo que generaría discapacidad permanente (30).

Delirium. El delirium conocido también como síndrome confusional agudo, se caracteriza por inatención, alteración en función cognoscitiva, psicomotora y curso fluctuante (32). Es un síndrome geriátrico frecuente en hospitalización, con prevalencia de $20 \%$ a $50 \%$ en servicios de hospitalización médicos y quirúrgicos y de $70 \%$ a $80 \%$ en unidades de cuidado intensivo (33).

Los ancianos admitidos a servicios de cuidado agudo con delirium, tienen mayor probabilidad de desarrollar desenlaces adversos como DF, cursar con enfermedad aguda más severa independientemente de la comorbilidad de base, desarrollar demencia uno a dos años después del egreso, requerir mayores cuidados, estancia hospitalaria y ser ingresados a un hogar de ancianos (34).

Las consecuencias funcionales adversas a largo plazo, se ven más acentuadas cuando el paciente presenta delirium al ingreso que cuando lo desarrolla durante la hospitalización. El delirium presente al momento de la admisión, tiene un impacto negativo en la capacidad funcional a los dos años similar al presentado en persona con fractura de cadera, con las mismas implicaciones en el funcionamiento cognoscitivo y afectivo, que llevan a una lenta recuperación y aumenta la probabilidad de dependencia en ABVD y AIVD (32). 
Condición funcional antes de hospitalización. Existe heterogeneidad con respecto al tiempo inicial y de seguimiento empleado para evaluar la DF. Algunos estudios evalúan la DF desde el estado premórbido mientras que otros lo evalúan desde la admisión hospitalaria (35). Los estudios antiguos conceptualizaron la DF de forma amplia, la relacionan con el episodio de enfermedad que llevó a la admisión hospitalaria, mientras que los estudios más recientes la asocian con resultados y eventos ocurridos durante la hospitalización (5). La distinción es importante porque la DF previa al ingreso muestra la condición de fragilidad siendo un predictor de desenlaces adversos incluido DF (29). En general, el estado funcional basal previo a hospitalización, se considera en la mayoría de las investigaciones como la condición funcional dos semanas antes del ingreso (5). Se han descrito como FR independientes para desarrollar DF al ingreso un Índice de Barthel (IB) menor de 60 (10), compromiso previo en AIVD (36) y el uso previo de ayudas para la movilidad (37).

Ambiente y rutinas hospitalarias. Aunque en la actualidad se dispone de instituciones hospitalarias modernas y con mejores equipos tecnológicos, muchas están diseñadas para el personal asistencial y cuidadores y no para los ancianos. Además, gran número de actividades asistenciales son realizadas según prácticas tradicionales sin la evidencia científica requerida (38). Por ejemplo, órdenes que limitan la movilidad, realización de exámenes o toma de signos vitales que alteran el descanso nocturno, empleo de líneas venosas y catéteres que predisponen a infecciones nosocomiales, restricciones físicas como sujeciones, barandas y las químicas como el empleo excesivo de psicofármacos, son algunas de los rutinas hospitalarias que se encuentran relacionadas con el desarrollo de DF $(20,28)$.

Con respecto a las condiciones arquitectónicas y diseño hospitalario, existen un gran número de barreras físicas que afectan la movilidad de los pacientes como las camas altas, habitaciones estrechas que limitan el empleo de ayudas para movilidad, sillones bajos, baños no adaptados, corredores poco iluminados y con obstáculos que incrementan el riesgo de caídas (5).

Edad. La edad es un factor asociado con el desarrollo de DF durante la hospitalización. Covinsky et al. documentaron que $23 \%$ de pacientes entre 70 y 74 años presentaron al alta alguna disminución en su situación funcional en comparación con el $50 \%$ en el grupo de los mayores de 85 años (4). Asimismo, Cruz et al. describen que 16\% de los menores de 85 años presentaban DF al alta frente al 67,5\% de los mayores de 85 años (39). Las anteriores investigaciones y la de Mañas et al. muestran que los mayores de 85 años presentan el mayor riesgo para desarrollar DF durante la hospitalización (40).

Compromiso cognoscitivo. Los ancianos hospitalizados con deterioro cognoscitivo de base presentan mayor riesgo para la DF. Lo anterior es explicado porque tienen mayores limitaciones para la movilidad, alteración comportamental con requerimiento de psicofármacos, inmovilización física, alteración del ciclo vigilia-sueño, que incrementan la probabilidad de presentar DF y síndromes geriátricos como caídas, delirium, incontinencia urinaria y fecal y úlceras por presión (41). El deterioro cognoscitivo al ingreso ha sido identificado en varios estudios como FR independiente para desarrollar DF al alta hospitalaria $(8,41)$.

Síntomas depresivos. La presencia de síntomas depresivos en ancianos hospitalizados se encuentra ente $10 \%$ y $25 \%$ según criterios diagnósticos empelados (42). Se ha evaluado la asociación entre síntomas depresivos y resultados funcionales en ancianos hospitalizados. Por ejemplo, Covinsky et al. documentaron que pacientes con 6 o más síntomas depresivos al ingreso tuvieron 3 veces mayor riesgo de DF, en comparación con los que solo tuvieron 2 o menos síntomas de depresión (42).

A pesar que los mecanismos fisiopatológicos no son bien conocidos, se considera que los síntomas negativos como apatía, anhedonia, anergia, hiporexia, alteración del ciclo circadiano, los efectos secundarios de los medicamentos y las enfermedades crónicas, generan un impacto negativo sobre el estado funcional durante la hospitalización (43).

Polifarmacia. Las reacciones medicamentosas pueden estar implicadas entre $20 \%$ y $25 \%$ en el desarrollo de la DF en ancianos hospitalizados (38). De igual manera el empleo de múltiples medicamentos se encuentra relacionado con mayor probabilidad de prescripciones inadecuadas y desarrollo de reacciones adversas por medicamentos (44), también la prescripción inadecuada en ancianos que viven en comunidad se relaciona con una peor condición funcional e ingresos hospitalarios (45). 
Evaluación del riesgo para declinación funcional

Cuadro 1. Características de las escalas de tamizaje para evaluar deterioro funcional asociado con hospitalización.

\begin{tabular}{|c|c|c|c|c|c|c|}
\hline Escala & Objetivo & $\begin{array}{l}\text { Lugar de } \\
\text { valoración }\end{array}$ & Variables & Puntuación & $\begin{array}{l}\text { Hazard } \\
\text { ratio } \\
\text { de DF }\end{array}$ & Aplicación \\
\hline HARP Sager & \multirow{5}{*}{$\begin{array}{l}\text { Estratificación del } \\
\text { riesgo para DF al alta y } \\
3 \text { meses }\end{array}$} & \multirow{5}{*}{$\begin{array}{l}\text { Salas de } \\
\text { hospitalización } \\
\text { médica }\end{array}$} & Edad $(0-1-2)$ & Rango: 0-5 & $17 \%$ & \\
\hline \multirow[t]{4}{*}{ et al. ${ }^{36}$} & & & $\begin{array}{l}\text { Deterioro cognitivo (MMSE } \\
\text { abreviado } 15 / 21)(0-1)\end{array}$ & & $28 \%$ & \\
\hline & & & $\begin{array}{l}\text { Dependencia previa AIVD ( } 7 \\
\text { funciones) } 2 \text { semanas antes de } \\
\text { hospitalización }(0-2)\end{array}$ & 0 -1: bajo riesgo & \multirow[t]{3}{*}{$56 \%$} & Personal clínico \\
\hline & & & & 2-3: intermedio & & \\
\hline & & & & 4-5: alto riesgo & & \\
\hline ISAR McCusker & \multirow{4}{*}{$\begin{array}{l}\text { I d e n t if i c a c i ó } \mathrm{n} \\
\text { de pacientes con } \\
\text { riesgo para muerte, } \\
\text { institucionalización y } \\
\text { DF, } 6 \text { meses posterior } \\
\text { de consulta a urgencias }\end{array}$} & & Dependencia funcional previa & Rango: 0 a 6 & \multirow{4}{*}{$\begin{array}{l}3.0 \\
(1,3- \\
6,94)\end{array}$} & \multirow{4}{*}{$\begin{array}{l}\text { Personal clínico } \\
\text { Autoadminis trada }\end{array}$} \\
\hline \multirow[t]{3}{*}{ et al. ${ }^{46}$} & & & $\begin{array}{l}\text { Cambio agudo en condición } \\
\text { funcional }\end{array}$ & & & \\
\hline & & Urgencias & $\begin{array}{l}\text { Hospitalización reciente } \\
\text { Problemas de memoria }\end{array}$ & $\begin{array}{l}\text { Sin puntos de } \\
\text { corte }\end{array}$ & & \\
\hline & & & $\begin{array}{l}\text { Problemas de visión Consumo } \geq \\
3 \text { fármacos }\end{array}$ & $\begin{array}{l}\text { Puntaje }>2 \\
\text { puntos indica } \\
\text { alto riesgo }\end{array}$ & & \\
\hline $\begin{array}{l}\text { SHERPA } \\
\text { Cornette }\end{array}$ & \multirow{6}{*}{$\begin{array}{l}\text { Estratificación del } \\
\text { riesgo para DF } 3 \text { meses } \\
\text { posterior del alta }\end{array}$} & Urgencias & Caída en el año último año (0-2) & Rango: 0-11.5 & & \multirow{6}{*}{ Personal clínico } \\
\hline \multirow[t]{5}{*}{ et al. ${ }^{1}$} & & $\begin{array}{l}\text { Salas de } \\
\text { hospitaliza- } \\
\text { ción médica }\end{array}$ & (MMSE abreviado 15/21) (0-2) & & & \\
\hline & & & Autopercepción de salud (0-1.5) & 0-3: bajo riesgo & 1 & \\
\hline & & & Edad $(0-1,5-3)$ & 3,5-4,5: leve & 2 & \\
\hline & & & $\begin{array}{l}\text { Dependencia previa para AIVD } \\
(0-1-2-3)\end{array}$ & $\begin{array}{l}\text { 5-6: moderado } \\
6-11,5: \text { alto }\end{array}$ & 4,2 & \\
\hline & & & & & 10,4 & \\
\hline TRST Hustey & \multirow{5}{*}{$\begin{array}{l}\text { Identificación del } \\
\text { riesgo de DF posterior } \\
\text { a consulta en urgencias } \\
\text { y hospitalización }\end{array}$} & \multirow{5}{*}{$\begin{array}{l}\text { Urgencias } \\
\text { Salas de } \\
\text { hospitaliza- } \\
\text { ción médica }\end{array}$} & $\begin{array}{l}\text { Deterioro cognitivo Dificultad } \\
\text { para caminar, transfe-rencias o } \\
\text { caídas recientes }\end{array}$ & De 0 a 6 & \multirow{5}{*}{$\begin{array}{l}2.58 \\
(1,5- \\
4,5)\end{array}$} & \multirow{5}{*}{$\begin{array}{l}\text { Personal clínico } \\
\text { Autoadminis-trada }\end{array}$} \\
\hline \multirow[t]{4}{*}{ et al..$^{47}$} & & & Vivir solo o sin cuidador & & & \\
\hline & & & Consumo habitual $>5$ fármacos & $\begin{array}{l}\text { Sin puntos de } \\
\text { corte }\end{array}$ & & \\
\hline & & & $\begin{array}{l}\text { Visita a urgencias en los últimos } \\
30 \text { días o ingreso en los últimos } \\
90 \text { días }\end{array}$ & & & \\
\hline & & & $\begin{array}{l}\text { Condición de riesgo según } \\
\text { enfermería }\end{array}$ & $\begin{array}{l}\text { Presencia } \\
\text { de deterioro } \\
\text { cognoscitivo } \\
\text { o puntaje }>2 \\
\text { puntos indica } \\
\text { alto riesgo }\end{array}$ & & \\
\hline
\end{tabular}

Modificada de Osuna-Pozo et al. ${ }^{5}$ 
HARP: Hospital admission risk profile, ISAR: Identification of Seniors At Risk o functional decline, SHERPA: Score Hospitalier d'Evaluation du Risque de Perte d'Autonomie, TRST: Triage Risk Screening Tool, MMSE: minimental test; DF: declinación functional; AIVD= actividades instrumentales de vida diaria.

Los estudios que han desarrollado escalas de tamizaje se han enfocado en la evaluación y predicción del riesgo global para DF más que en los FR individuales (27). Según su diseño, las escalas se pueden aplicar en servicios de urgencias o en hospitalización, se caracterizan por ser breves y de fácil realización $(46,47)$. Las más empleadas son el HARP (36), ISAR (46), SHERPA (1) y TRST (47) (Cuadro 1).

Se han propuesto recomendaciones para mejorar las investigaciones que desarrollan escalas de predicción para DF (48). Inicialmente se requiere determinar el objetivo buscado con su aplicación, por ejemplo, intervenir sobre FR o evaluar el riesgo de ser institucionalizado posterior al alta, porque no siempre estos dos factores implican el desarrollo de desenlaces similares $(24,27)$. Después establecer quién, dónde y cuándo realizará su aplicación (urgencias, unidad de agudos, cuidado intensivo), en cualquiera de estas se debe practicar antes de las primeras 48 horas del ingreso (5). Con respecto a la escala a evaluar se sugiere que esté validada, de uso estandarizado y los diferentes FR a evaluar reflejen puntuación proporcional según su importancia. Finalmente, se sugiere incluir en las nuevas escalas, marcadores clínicos de fragilidad como la fuerza de agarre, velocidad de la marcha y algunos marcadores biológicos como proteína $\mathrm{C}$ reactiva, interleucina 6 y factor de necrosis tumoral (29).

Manejo de declinación funcional

Luego de la identificación del anciano con riesgo o DF, se deben llevar a cabo intervenciones para su prevención y manejo, dentro de las cuales se tienen:

Intervenciones basadas en ejercicio. En ancianos hospitalizados el entrenamiento físico de miembros inferiores puede preservar o mejorar la condición funcional, movilidad y marcha. Lo anterior es función más de la intensidad del ejercicio que de otras variables como la edad, el sexo o condición de salud (49).

La colaboración Cochrane en 2007 publicó una revisión de investigaciones sobre el efecto del ejercicio en ancianos hospitalizados por enfermedad médica aguda (50). Se seleccionaron estudios que evaluaran el efecto del ejercicio solo o asociado con una intervención geriátrica multidimensional, comparado con la atención convencional. Los estudios mostraron diferencias en aspectos metodológicos e intervenciones que dificultó el análisis, comparación y validez de los resultados, además solo cinco investigaciones tuvieron información sobre el estado funcional al alta (23,51-54).

En las investigaciones de Landefeld et al (51). y de Counsell et al. (52), la intervención geriátrica fue similar, se aplicó mediante un modelo de atención especializado con el anciano basado en adaptación del entorno, atención centrada en el anciano, revisión médica diaria, protocolos para prevención de síndromes geriátricos y egreso geriátrico planeado.

El estudio de Slaets et al. la intervención geriátrica fue realizada por un psicogeriatra que lideraba el equipo, enfermera de enlace, fisioterapeuta y 3 enfermeras más que la atención convencional (23). La intervención consistió en fisioterapia y rehabilitación para prevención de DF, pero no se especificó cómo se realizó. El grupo que recibió programa de ejercicio consistió en caminar o ponerse en pie 3 veces al día e ir caminando en la sala realizando actividades.

Las tres investigaciones que aplicaron intervención geriátrica multidimensional con ejercicio, obtuvieron mejores resultados funcionales durante la hospitalización comparado con la atención convencional $(23,51,52)$ y las dos investigaciones donde solo se realizó ejercicio hubo una tendencia hacia la mejoría $(53,54)$. Sin embargo, se requieren de más estudios con adecuado diseño metodológico para evaluar el efecto del programa de ejercicio en ancianos hospitalizados, además de describir de forma adecuada las intervenciones.

Bachmann et al. realizaron una revisión sistemática y meta análisis sobre programas de rehabilitación hospitalaria en población geriátrica (55). Incluyeron 17 ensayos clínicos aleatorizados que evaluaron el efecto en una unidad geriátrica que incluían rehabilitación (fisioterapia, terapia ocupacional o ambas) comparado con la atención convencional; se analizaron por aparte los programas de rehabilitación geriátrica y los de fractura de cadera. Se documentaron resultados favorables en términos de mejoría funcional, menor institucionalización y mortalidad, tanto al egreso como al seguimiento. En el grupo de fractura de cadera hubo mejoría funcional.

Cuidado geriátrico especializado. El manejo de ancianos hospitalizados en UGA comparado con la atención hospitalaria convencional, ha mostrado beneficios que incluyen mejoría del estado funcional, menor probabilidad de institucionalización sin incrementar los costos de atención (56).

En este modelo se realizan intervenciones multicomponente que permiten disminuir los efectos adversos de la hospitalización y mejorar la condición funcional. Las intervenciones están dirigidas a modificar el entorno, cuidado centrado en el anciano, asegurar la adherencia al tratamiento, protocolos para prevenir y rehabilitar pacientes con DF y diseño del egreso geriátrico planeado $(52,56)$. Teóricamente el modelo puede ser implementado en cualquier unidad hospitalaria que atiende ancianos, pero requeriría de las modificaciones del entorno y la presencia del equipo interdisciplinario que disponga de un líder que pertenezca al equipo (57).

El equipo interdisciplinario determina la trayectoria funcional para la condición del anciano durante la hospitalización, en la transición entre el egreso y el destino (hogar, institucionalización), además de los requerimientos domiciliarios (servicios de cuidados de enfermería, rehabilitación, etc.), con el objetivo que sea un retorno positivo al domicilio $(4,35)$.

\section{Conclusiones}

La DF asociada con hospitalización es un evento de ocurrencia frecuente que refleja la morbilidad esperada debido a los cambios anatómicos y fisiológicos que genera el envejecimiento, fragilidad y la propia enfermedad aguda. Afecta la calidad de vida e independencia de los ancianos, cuidadores e incrementa los costos en atención de salud. Se puede desarrollar antes, durante y posterior a la hospitalización. La identificación oportuna es el primer paso para la valoración del riesgo y determinar los FR con el objeto de prevenir y manejar esta condición. Los clínicos deberían modificar el cuidado hospitalario tradicional enfocado en la enfermedad por una aproximación integral donde incluya y reconozca al estado funcional como un signo clínico vital que refleja la manifestación de la enfermedad aguda durante y después de la hospitalización. En ancianos con enfermedad aguda, el manejo por un equipo interdisciplinario en una UGA, mediante intervenciones 
dirigidas a modificación del entorno, cuidado centrado en el anciano, revisión del manejo farmacológico, programas de ejercicio y diseño del egreso geriátrico planeado, ha mostrado tener buenos resultados en la prevención y/o manejo de la DF. La implementación de modelos de atención que prevengan y mejoren la DF deber ser una prioridad para las instituciones y personal de salud que atienden ancianos.

\section{Referencias}

1. Cornette P, Swine C, Malhomme B, Gillet JB, Meert P, D'Hoore W. Early evaluation of the risk of functional decline following hospitalization of older patients: development of a predictive tool. Eur J Public Health. 2006; 16: 203-8.

2. Baztán J, Suárez F, García F. Informe sobre la necesidad de unidades geriátricas de agudos y unidades geriátricas de recuperación funcional como parte de los recursos hospitalarios especializados para personas mayores. Madrid: SEMEG 2004.

3. Thomas DR. Guest Editorial: focus on functional decline in hospitalized older adults. J Gerontol Series A: Biol Sci Med Sci. 2002; 57: M567-M8.

4. Covinsky KE, Palmer RM, Fortinsky RH, Counsell SR, $\underline{\text { Stewart }}$ AL, Krevevic D, et al. Loss of independence in activities of daily living in older adults hospitalized with medical illnesses: increased vulnerability with age. J Am Geriat Soc. 2003; 51: 451-8.

5. Osuna-Pozo CM, Ortiz-Alonso J, Vidán M, Ferreira G, SerraRexach JA. Revisión sobre el deterioro funcional en el anciano asociado al ingreso por enfermedad aguda. Rev Español Geriatr Gerontol. 2014; 49: 77-89.

6. Boyd M, Koziol-McLain J, Yates K, Kerse N, McLean C, Pilcher C, et al. Recovery of activities of daily living in older adults after hospitalization for acute medical illness. J Am Geriatr Soc. 2008; 56: 2171-9.

7. Zisberg A, Shadmi E, Sinoff G, Gur-Yaish N, Srulovici E, Admi $\mathrm{H}$. Low mobility during hospitalization and functional decline in older adults. J Am Geriatr Soc. 2011; 59: 266-73.

8. Sager MA, Franke T, Inouye SK, Landefeld CS, Morgan TM, Rudberg MA, et al. Functional outcomes of acute medical illness and hospitalization in older persons. Arch Intern Med. 1996; 156: 645-52.

9. Margitic SE, Inouye SK, Thomas JL, Cassel CK, Regenstreif DI, Kowal J. Hospital Outcomes Project for the Elderly (HOPE): rationale and design for a prospective pooled analysis. J Am Geriatr Soc. 1993; 41: 258-67.

10. Abizanda Soler P, León Ortiz M, Romero Rizos L, Sánchez Jurado PM, Luengo Márquez C, Domínguez Martín L, et al. La pérdida funcional al ingreso, principal variable explicativa de discapacidad y mortalidad al alta y al mes en ancianos hospitalizados. Rev Español Geriatr Gerontol. 2007; 42: 201-11.

11. Zelada MA, Salinas R, Baztan JJ. Reduction of functional deterioration during hospitalization in an acute geriatric unit. Arch Gerontol Geriatr. 2009; 48: 35-9.

12. Verbrugge LM, Jette AM. The disablement process. Soc Sci Med. 1994; 38: 1-14.

13. Nagi S. Disability concepts revisited: implications for prevention. In: Pope A, Tarlov A, eds. Disability in America: Toward a National Agenda for Prevention. Washinton, DC: National Academy Press; 1991: 309-27.

14. Ocampo JM. Self-rated health: Importance of use in elderly adults. Colomb Med. 2010; 41: 275-89.

15. Reyes-Ortiz CA. The disability-poverty connection in older people. J Natl Med Assoc. 1999; 91: 75-7.
16. Palmer RM, Counsell S, Landefeld CS. Clinical intervention trials: the ACE unit. Clin Geriatr Med. 1998; 14: 831-49.

17. Creditor MC. Hazards of hospitalization of the elderly. Ann Intern Med. 1993; 118: 219-23.

18. Sullivan DH, Sun S, Walls RC. Protein-energy undernutrition among elderly hospitalized patients: a prospective study. JAMA. 1999; 281: 2013-9.

19. Ocampo J. Delirium inducido por medicamentos. In: Marulanda F, Ocampo J, Osorno D, eds. Delirium: un gigante de la geriatría. Manizales: Editorial Universidad de Caldas; 2009: 213-22.

20. Covinsky KE, Pierluissi E, Johnston CB. Hospitalizationassociated disability: She was probably able to ambulate, but I'm not sure. JAMA. 2011; 306: 1782-93.

21. Alarcón T, Barcena A, González-Montalvo JI, Peñalosa C, Salgado A. Factors predictive of outcome on admission to an acute geriatric ward. Age Ageing. 1999; 28: 429-32.

22. Sleiman I, Rozzini R, Barbisoni P, Morandi1 A, Riccil A,Giordano A, Trabucchi M. Functional trajectories during hospitalization: a prognostic sign for elderly patients. J Gerontol A Biol Sci Med Sci. 2009; 64: 659-63.

23. Slaets JP, Kauffmann RH, Duivenvoorden HJ, Pelemans W, Schudel WJ. A randomized trial of geriatric liaison intervention in elderly medical inpatients. Psychosom Med. 1997; 59: 585-91.

24. Rudberg MA, Sager MA, Zhang J. Risk factors for nursing home use after hospitalization for medical illness. J Gerontol A Biol Sci Med Sci. 1996; 51: M189-94.

25. Inouye SK, Studenski S, Tinetti ME, Kuchel GA. Geriatric syndromes: clinical, research, and policy implications of a core geriatric concept. J Am Geriatr Soc. 2007; 55: 780-91.

26. Reyes-Ortiz CA. Urinary incontinence in elderly patients. JAMA. 1997; 277: 881-2.

27. McCusker J, Kakuma R, Abrahamowicz M. Predictors of functional decline in hospitalized elderly patients a systematic review. J Gerontol Series A: Biol Sci Med Sci. 2002; 57: M569-M77.

28. de Saint-Hubert M, Schoevaerdts D, Poulain G, Cornette P, Swine C. Risk factors predicting later functional decline in older hospitalized patients. Acta Clin Belg. 2009; 64: 187-94.

29. Lafont C, Gerard S, Voisin T, Pahor M, Vellas B. Reducing "iatrogenic disability" in the hospitalized frail elderly. J Nutr Health Aging. 2011; 15: 645-60.

30. Kortebein P, Ferrando A, Lombeida J, Wolfe R, Evans WJ. Effect of 10 days of bed rest on skeletal muscle in healthy older adults. JAMA. 2007; 297: 1772-4.

31. Brown CJ, Redden DT, Flood KL, Allman RM. The underrecognized epidemic of low mobility during hospitalization of older adults. J Am Geriatr Soc. 2009; 57: 1660-5.

32. Inouye SK. Delirium in older persons. N Engl J Med. 2006; 354: 1157-65.

33. Inouye SK, Westendorp RG, Saczynski JS. Delirium in elderly people. Lancet. 2014; 383: 911-22.

34. Siddiqi N, House AO, Holmes JD. Occurrence and outcome of delirium in medical in-patients: a systematic literature review. Age Ageing. 2006; 35: 350-64.

35. Covinsky KE, Palmer RM, Counsell SR, Pine ZM, Walter LC, Chren MM. Functional status before hospitalization in acutely ill older adults: validity and clinical importance of retrospective reports. J Am Geriatr Soc. 2000; 48: 164-9.

36. Sager MA, Rudberg MA, Jalaluddin M, Franke T, Inouye SK, Landefeld CS, et al. Hospital admission risk profile (HARP): identifying older patients at risk for functional decline following acute medical illness and hospitalization. J Am Geriatr Soc. 1996; 44: 251-7. 
37. Hoogerduijn JG, Buurman BM, Korevaar JC, Grobbee DE, de Rooij SE, Schuurmans MJ. The prediction of functional decline in older hospitalised patients. Age Ageing. 2012; 41: 381-7.

38. Sager MA, Rudberg MA. Functional decline associated with hospitalization for acute illness. Clin Geriatr Med. 1998; 14: 66979.

39. Cruz Lendínez AJ, Pancorbo Hidalgo PL, García Fernández FP, Carrascosa García MI, Díaz J, Villar Dávila R. Deterioro funcional en ancianos ingresados en un hospital sin unidades geriátricas. Gerokomos. 2010; 21: 8-16.

40. Mañas MD, Marchan E, Conde C, Sánchez S, Sánchez-Maroto T, Molina MC. Functional impairment in elderly patients hospitalised in an Internal Medicine unit. An Med Internal. 2005; 22: $130-2$.

41. Pedone C, Ercolani S, Catani M, Maggio D, Ruqqiero C, Quartesan R, et al. Elderly patients with cognitive impairment have a high risk for functional decline during hospitalization: The GIFA Study. J Gerontol A Biol Sci Med Sci. 2005; 60: 1576-80.

42. Covinsky KE, Fortinsky RH, Palmer RM, Kresevic DM, Landefeld CS. Relation between symptoms of depression and health status outcomes in acutely ill hospitalized older persons. Ann Intern Med. 1997; 126: 417-25.

43. Taylor WD. Clinical practice. Depression in the elderly. $N$ Engl $J$ Med. 2014; 371: 1228-36.

44. Delgado Silveira E, Muñoz García M, Montero Errasquin B, Sánchez Castellano C, Gallagher PF, Cruz-Jentoft AJ. Inappropriate prescription in older patients: the STOPP/START criteria. Rev Esp Geriatr Gerontol. 2009; 44: 273-9.

45. Corsonello A, Pedone C, Lattanzio F, Lucchetti M, Garasto S, Di Muzio M, et al. Potentially inappropriate medications and functional decline in elderly hospitalized patients. J Am Geriatr Soc. 2009; 57: 1007-14.

46. McCusker J, Bellavance F, Cardin S, Trepanier S, Verdon J, Ardman O. Detection of older people at increased risk of adverse health outcomes after an emergency visit: the ISAR screening tool. J Am Geriatr Soc. 1999; 47: 1229-37.

47. Hustey FM, Mion LC, Connor JT, Emerman CL, Campbell J, Palmer RM. A brief risk stratification tool to predict functional decline in older adults discharged from emergency departments. J Am Geriatr Soc. 2007; 55: 1269-74.

48. De Saint-Hubert M, Schoevaerdts D, Cornette P, D'Hoore W, Boland B, Swine C. Predicting functional adverse outcomes in hospitalized older patients: a systematic review of screening tools. J Nutr Health Aging. 2010; 14: 394-9.

49. Suetta C, Magnusson SP, Beyer N, Kjaer M. Effect of strength training on muscle function in elderly hospitalized patients. Scand J Med Sci Sports. 2007; 17: 464-72.

50. de Morton NA, Keating JL, Jeffs K. Exercise for acutely hospitalised older medical patients. Cochrane Database Syst Rev. 2007: Cd005955.

51. Landefeld CS, Palmer RM, Kresevic DM, Fortinsky RH, Kowal J. A randomized trial of care in a hospital medical unit especially designed to improve the functional outcomes of acutely ill older patients. N Engl J Med. 1995; 332: 1338-44.

52. Counsell SR, Holder CM, Liebenauer LL, Palmer RM, Fortinsky $\mathrm{RH}$, Kresevic DM, et al. Effects of a multicomponent intervention on functional outcomes and process of care in hospitalized older patients: a randomized controlled trial of Acute Care for Elders (ACE) in a community hospital. J Am Geriatr Soc. 2000; 48: 157281.
53. de Morton NA, Keating JL, Berlowitz DJ, Jackson B, Lim WK. Additional exercise does not change hospital or patient outcomes in older medical patients: a controlled clinical trial. Aust $J$ Physiother. 2007; 53: 105-11.

54. Jones CT, Lowe AJ, MacGregor L, Brand CA, Tweddle N, Russell DM. A randomised controlled trial of an exercise intervention to reduce functional decline and health service utilisation in the hospitalised elderly. Austral J Ageing. 2006; 25: 126-33.

55. Bachmann S, Finger C, Huss A, Egger M, Stuck AE, Clough-Gorr KM. Inpatient rehabilitation specifically designed for geriatric patients: systematic review and meta-analysis of randomised controlled trials. BMJ. 2010; 340: c1718.

56. Baztan JJ, Suárez-García FM, López-Arrieta J, Rodríguez-Manas L, Rodríguez-Artalejo F. Effectiveness of acute geriatric units on functional decline, living at home, and case fatality among older patients admitted to hospital for acute medical disorders: metaanalysis. BMJ. 2009; 338: b50.

57. Fox MT, Persaud M, Maimets I, O’Brien K, Brooks D, Trequnno $\mathrm{D}$, et al. Effectiveness of acute geriatric unit care using acute care for elders components: a systematic review and meta-analysis. $J$ Am Geriatr Soc. 2012; 60: 2237-45. 